\title{
Erythrocyte Toxicities of Imidazolidinyl Urea and Diazolidinyl Urea
}

\author{
Halla Noureddine, Boucherit Kebir, Boucherit Zahia and Rahmoun Nadjib \\ Antibiotics Antifungal Laboratory, Physical Chemistry, Synthesis and Biological Activity, Department of Molecular and Cellular \\ Biology, Abou Bekr Belkaïd University, Tlemcen 13000, Algeria
}

Received: December 23, 2012 / Accepted: January 27, 2013 / Published: July 25, 2013.

\begin{abstract}
The addition of antimicrobial preservatives to pharmaceutical and cosmetic products is necessary to prevent microbial growth. However, the use of preservatives can also produce other undesirable effects. For several years, researchers have been investigating the use of alternative methods in safety assessment of cosmetic ingredients and formulations by means of variety methods. The aim of this study was to evaluate the erythrocyte toxicities of two commercial preservatives: imidazolidinyl urea and diazolidinyl urea. Relatively few studies about the cytotoxicity of these preservative are available. The determination of their cytotoxicity is an essential step to warrant their safe use. Erythrocyte toxicities were evaluated by assessment of the amount of hemoglobin released by red blood cells after their lysis. In this study, both imidazolidinyl urea and diazolidinyl urea showed cytotoxic activity against red blood cells. The imidazolidinyl urea induce a small release of hemoglobin after 120 min of incubation. But, the diazolidinyl urea induce a massive release of hemoglobin from the imidazolidinyl urea (a rate of $83 \%$ at concentrations of $6.25 \mathrm{mg} / \mathrm{mL}$ and $12.5 \mathrm{mg} / \mathrm{mL})$.
\end{abstract}

Key words: Erythrocyte toxicity, imidazolidinyl urea, diazolidinyl urea, preservative, cosmetic.

\section{Introduction}

The safety of workers and consumers of cosmetic products has demanded the acceleration of research on the toxicity of their ingredients. But, the legislation has limited the toxicity tests realized on animals. Several in vitro systems for predicting the effect of toxics, as an alternative to animal tests, have been developed [1-4]. In fact, the use of alternative methods as non-animal tests, to evaluate the safety of chemical agents has become imperative in the recent years $[5,6]$.

Since the development of the hemolytic method by Husa and co-workers [7, 8], many research teams have used this method to evaluate the erythrocyte toxicity of various compounds.

A special class of preservatives is known as formaldehyde releasers agents, and they may exhibit

Corresponding author: Halla Noureddine, Ph.D. student, research fields: biological activity of synthetic or natural compounds. E-mail: halla.nour@yahoo.fr. their action by releasing formaldehyde or by the action of the parent chemical structure of the compounds. The most prominent of these compounds are: imidazolidinyl urea (IU) and diazolidinyl urea (DU) [9]. The two preservatives have been used as additives alone or in combination with other preservatives worldwide [10, 11]. According to the European Cosmetic Products Directive (Annex VI), the maximum authorized concentration of IU in cosmetic products is $0.6 \%$ [12]; while, the DU is recommended to be used in the range of $0.1 \%$ to $0.3 \%$ in topical preparations [13].

Imidazolidinyl urea (Gennall 115) was described in 1970 as antimicrobial preservatives [14] in cosmetic products such as mascaras and eyeliners [15]. It has since become the second most widely used preservative in the cosmetic industry after the parabens [16].

A safety assessment of Imidazolidinyl urea was published in 1980 with the conclusion that this 
ingredient is "safe when incorporated in cosmetic products in amounts similar to those presently marketed" [17]. In 2001, the Cosmetic Ingredient Review (CIR) Expert Panel found no new data contradicting the original conclusions in the imidazolidinyl urea safety assessment conducted in 1980 [18].

Imidazolidinyl urea induced a significant dose- and time-dependent decrease in cell viability of HL60 cells after 3,6 , or $24 \mathrm{~h}$ of incubation at a concentration range of $0.01 \%-1 \%$ [19]. It may also cause contact dermatitis. There is a lack of information on the cytotoxicity of this compound to which humans are widely exposed.

Diazolidinyl urea was marketed in 1982 by Sutton Laboratories under the name Germall II [13]. diazolidinyl urea (DU) is used as an antimicrobial preservative in many topical pharmaceutical and cosmetic formulations [20]. DU is known to be the most active member of the Imidazolidinyl urea family of preservatives.

DU shows a high sensitizing potential and many cases of contact dermatitis have been described in the literature [21, 22]. The Cosmetic Ingredient Review (CIR) Expert Panel confirmed that DU is safe up to a maximum concentration of $0.5 \%$ [23]. Although DU liberates formaldehyde, the data found in the literature do suggest that it is not mutagenic in the Salmonella typhimurium mutagenicity assay [24].

Clearly, very few studies of the toxicity of IU and DU have been done, and none of them were erythrocyte cytotoxicity studies.

During this work, we tried to set up red blood cells of human and then to test the cytotoxicity of the two preservatives IU and DU used in the cosmetic products.

We tried to determine the toxicity of a range of increasing concentrations with an arbitrary contact time.

\section{Experiments}

\subsection{Preservative Agents}

Imidazolidinyl urea and diazolidinyl urea (Sigma,
USA) were used for erythrocyte cytotoxicity studies. The appropriate weight of each preservative was added to $1 \mathrm{~L}$ of distillate water. The solutions were filter-sterilized and $100 \mathrm{~mL}$ of test solution dispensed into $250 \mathrm{~mL}$ flasks. The test solutions were used extemporaneously after their preparations.

\subsection{Isolation of Human Erythrocytes}

The erythrocyte toxicity assay was conducted as described by Bolard [25]. Freshly collected blood was used to assess the erythrocyte toxicities of the imidazolidinyl urea and diazolidinyl urea. Erythrocytes were centrifuged in heparinized tube at 4,000 rpm for 5 min. After removal of supernatant, the pellet was washed twice with the washing solution of magnesium chloride $\mathrm{MgCl}_{2}(2 \mathrm{mM})$ containing $150 \mathrm{mM} \mathrm{NaCl}$, then resuspended again in phosphate-buffered saline sodium (PBS) $100 \mathrm{mM}, \mathrm{pH}$ 7.4, containing also 150 $\mathrm{mM} \mathrm{NaCl}$.

\subsection{Measurement of Erythrocyte Toxicity}

Red blood cells were suspended in PBS pH 7.4, 100 $\mathrm{mM}$, at a rate of 4,000 cells $/ \mathrm{mL}$. The erythrocyte suspension was incubated at $37{ }^{\circ} \mathrm{C}$ under continuous agitation for $120 \mathrm{~min}$, from the addition of the preservative solution at different final concentrations. Samples of $500 \mu \mathrm{L}$ from the reaction solution were made at regular intervals to which we added $2 \mathrm{~mL}$ of cold washing solution (150 $\mathrm{mM} \mathrm{NaCl}, 2 \mathrm{mM} \mathrm{MgCl} 2)$. After centrifugation at $4,000 \mathrm{rpm}$ for $5 \mathrm{~min}$, the absorption of the resulting supernatant was determined at $548 \mathrm{~nm}$ by photometric monitoring against a blank sample. Control samples of $0 \%$ lysis (in buffer) and $100 \%$ lysis (in double-distilled water) were employed in all experiments $[25,26]$.

\section{Results and Discussion}

Erythrocyte-induced hemolysis in vitro can be considered to be a simple and reliable measure for estimating the membrane damage caused in vivo [27, 28]. Therefore, the effects of IU and DU in vivo were 
predicted by investigating the degree of hemolysis in vitro.

Our work focused on evaluating the cytotoxicity of the two preservatives: imidazolidinyl urea and diazolidinyl urea against the human red blood cells. When the plasma membrane of red blood cell was affected by the action of the preservatives, it followed that lysis results in release of hemoglobin from red blood cells, so we measured the extracellular hemoglobin after adding different concentrations of preservatives. The final concentrations of each preservative were chosen based on the maximum concentrations used in cosmetic products.

Figs. 1 and 2 represent the effect of imidazolidinyl urea and diazolidinyl urea, respectively, prepared in concentrations from 0.097 to $12.5 \mathrm{mg} / \mathrm{mL}$ on the release of hemoglobin from red blood cells at $37^{\circ} \mathrm{C}$. The control curve represents the curve where there was no addition of preservative; we do not hemoglobin in the extracellular medium during the $120 \mathrm{~min}$ of incubation.

\subsection{Effect of Imidazolidinyl Urea (IU)}

Figs. 1a and $1 \mathrm{~b}$ represent the effect of imidazolidinyl urea prepared at concentrations of 0.097 to 0.195 $\mathrm{mg} / \mathrm{mL}$. After $90 \mathrm{~min}$ of incubation, red cells lost around $5 \%$ of their hemoglobin. This loss was about $10 \%$ after $120 \mathrm{~min}$ at these concentrations. Figs. $1 \mathrm{c}-1 \mathrm{~h}$ represent the effect of imidazolidinyl urea prepared at concentrations from 0.39 to $12.5 \mathrm{mg} / \mathrm{mL}$. The extracellular hemoglobin was around $10 \%$ after $5 \mathrm{~min}$ of incubation and remained constant during the first 90 min of incubation for all concentrations. After $120 \mathrm{~min}$ of incubation, the rate reached a percentage of $20 \%$ to $33 \%$.

The maximum authorized concentration of IU in cosmetic products is $0.6 \%$ [29]; this concentration induced the release of hemoglobin from red cells to the rate of: $33 \%-36 \%$ after 120 min of incubation.

The IU product consists of at least 4 compounds: compound (4-hydroxymethyl-2,5-dioxo-imidazolidin- 4-yl)-urea, allantoin and two unidentified minor presumably formaldehyde-releasing compounds. To an unknown extent also the polymeric condensation products may release formaldehyde [9].

According to the results, we noted that the hemoglobin was constant during 90 min of incubation; it increased after $120 \mathrm{~min}$ of incubation at hemoglobin levels doubled.

It seemed that in the first time, imidazolidinyl urea caused lysis of red blood cells by the release of formaldehyde in aqueous medium. Formaldehyde was a component that was small, highly reactive and had an effect that translated immediately after contact with these targets [30]. In the second step, we could assume that the imidazolidinyl urea manifested its cytotoxicity by these parent molecules that could show that their activities slowly released formaldehyde.

\subsection{Effect of Diazolidinyl Urea (DU)}

Figs. 2a and 2e represent the effect of diazolidinyl urea prepared at concentrations of 0.097 to $1.562 \mathrm{mg} / \mathrm{mL}$. Red blood cells lost nearly $10 \%$ of their hemoglobin after 5 min of incubation. Hemolysis was estimated at $35 \%$ to $62 \%$ after 120 min of incubation. Fig. 2f represents the effect of Diazolidinyl urea to $3.125 \mathrm{mg} / \mathrm{mL}$. Red blood cells lost almost $5 \%$ of their hemoglobin after $5 \mathrm{~min}$ of incubation and nearly $30 \%$ after 20 min of incubation and remained constant until 40 min of incubation. This loss was around $74 \%$ after $120 \mathrm{~min}$ of incubation. Figs. $2 \mathrm{~g}$ and $2 \mathrm{~h}$ represent the effect of Diazolidinyl urea 6.25 and $12.5 \mathrm{mg} / \mathrm{mL}$. We noticed a $20 \%$ loss of hemoglobin after $5 \mathrm{~min}$ of incubation. This rate remained constant during $30 \mathrm{~min}$ of incubation. After $40 \mathrm{~min}$ of incubation, the releases of hemoglobin increase over time and reached a rate of $83 \%$ at 120 min of incubation.

The maximum authorized concentration of DU in cosmetic products is $0.5 \%$ [29]; this concentration induced the release of hemoglobin from red cells to the rate of $75-82 \%$ after 120 min of incubation. 


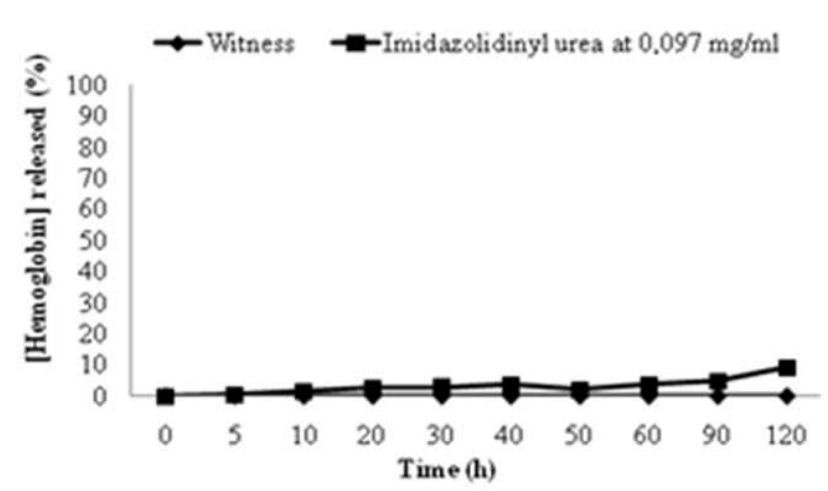

(a)

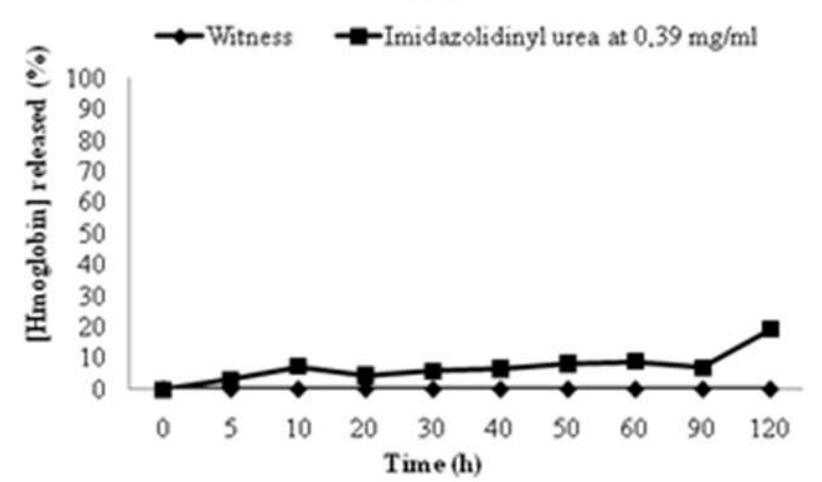

(c)

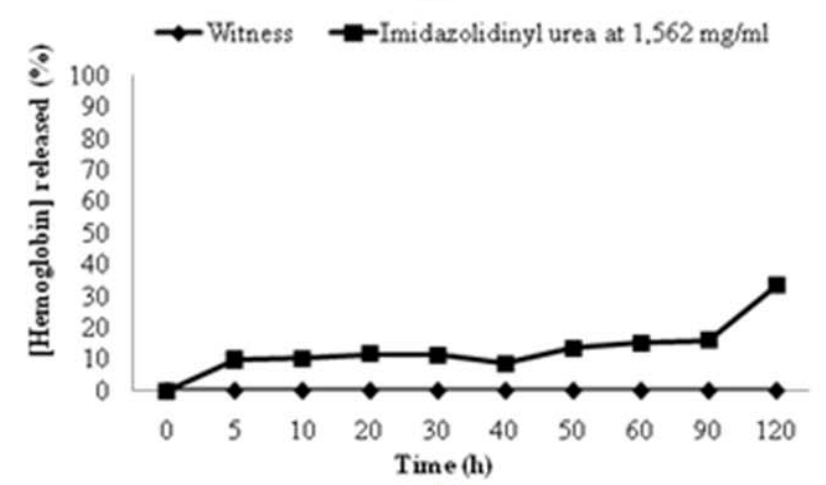

(e)

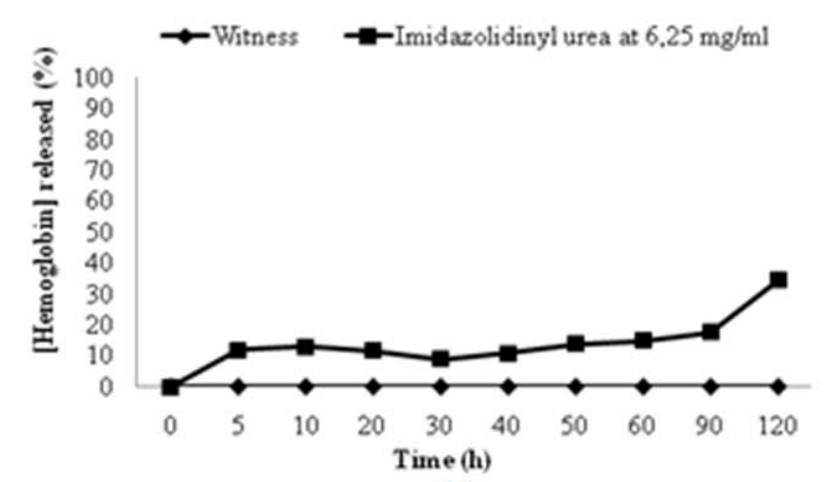

(g)

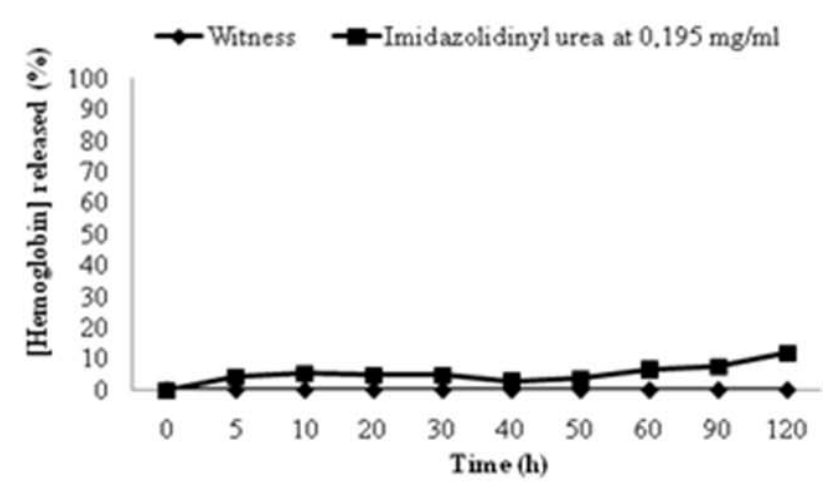

(b)

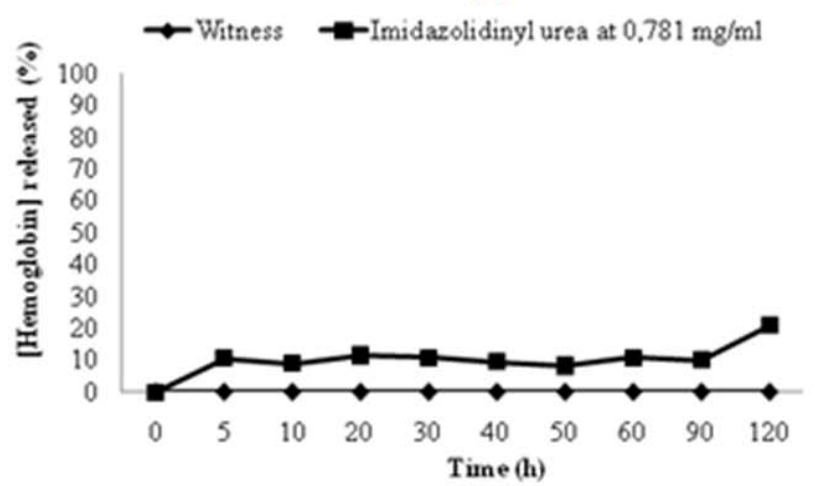

(d)

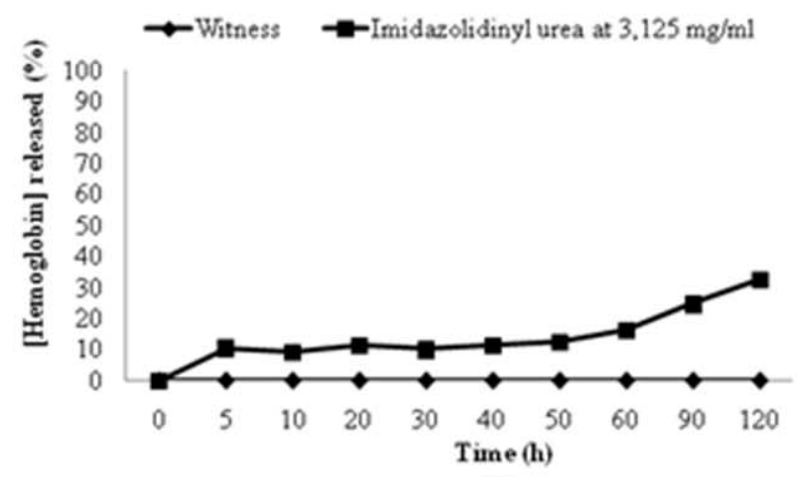

(f)

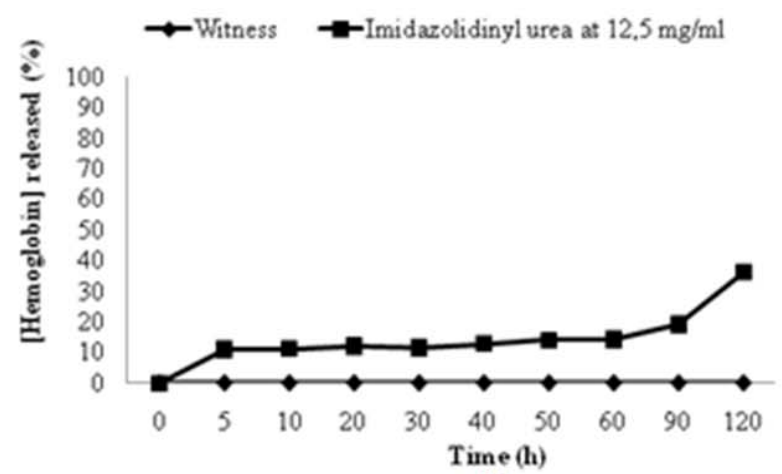

(h)

Fig. 1 Release of hemoglobin by erythrocytes induced by different concentrations of Imidazolidinyl urea (a) 0.097, (b) 0.195, (c) 0.39, (d) 0.781, (e) 1.562, (f) 3.125, (g) 6.25 and (h) $12.5 \mathrm{mg} / \mathrm{mL}$. 


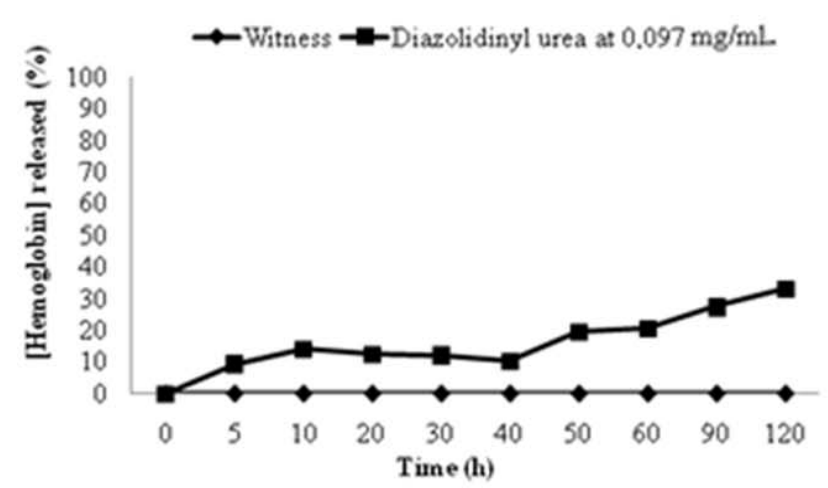

(a)

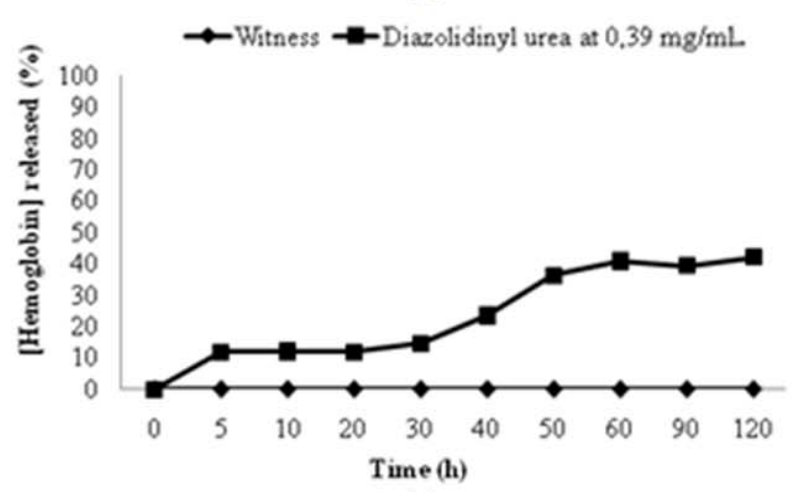

(c)

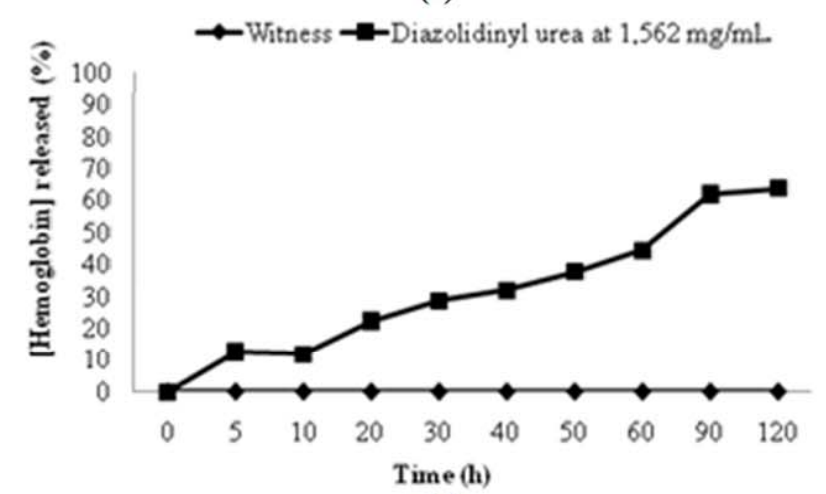

(e)

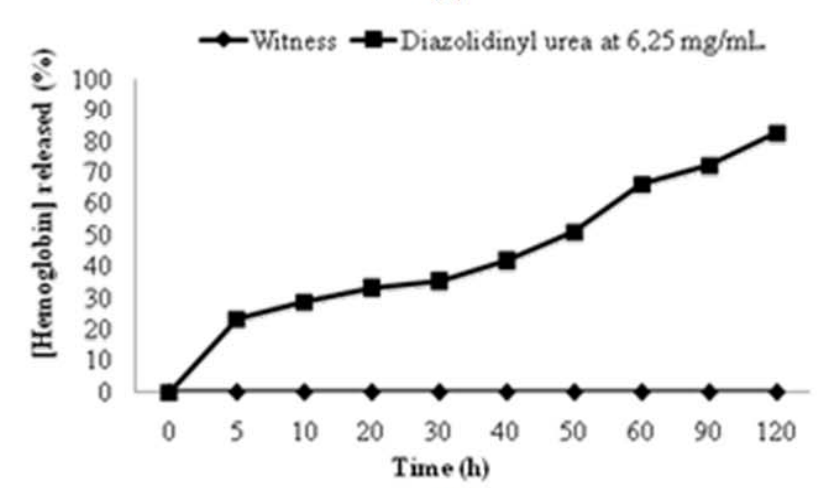

(g)

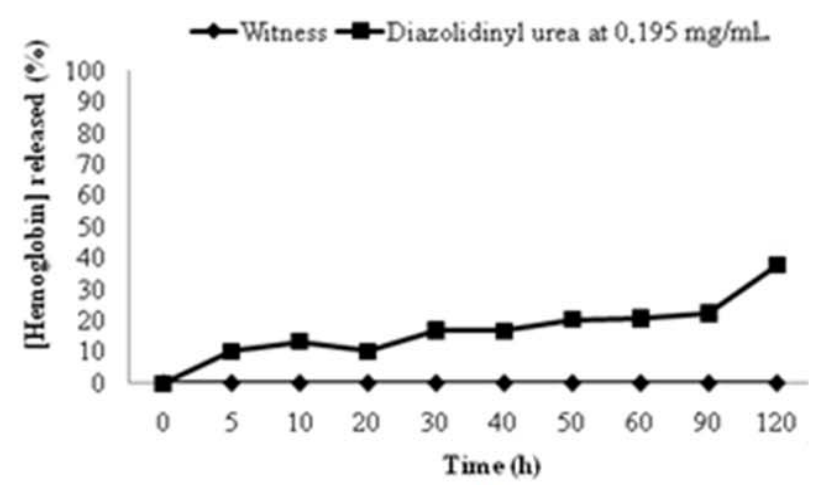

(b)

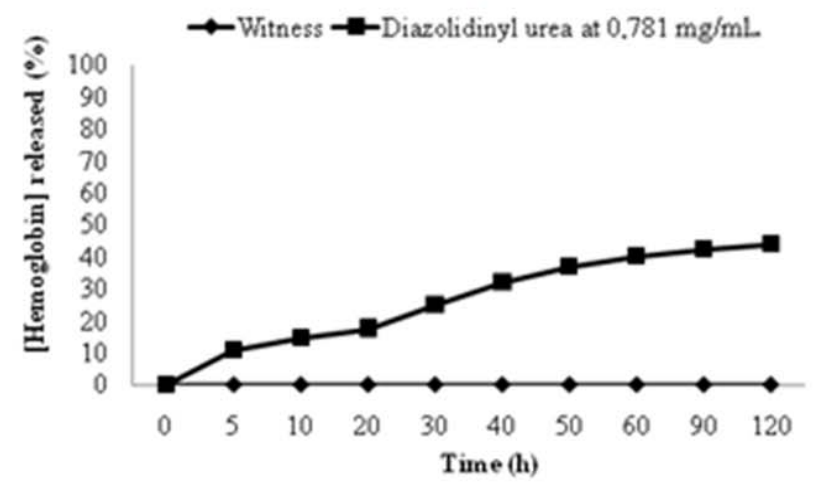

(d)

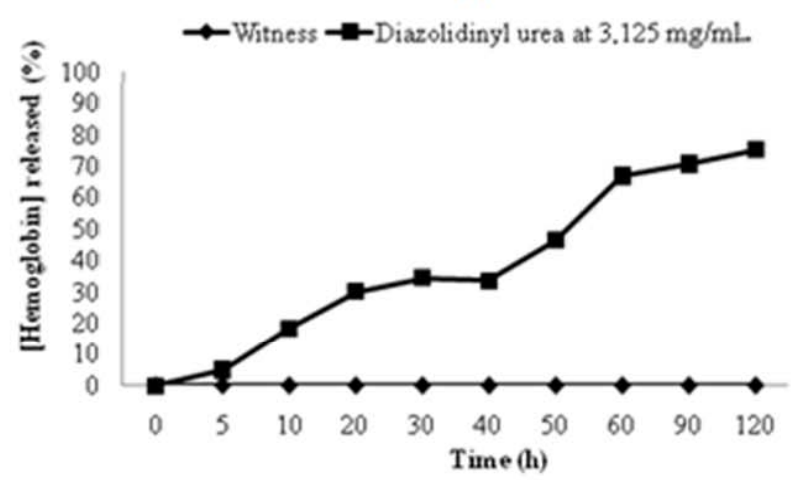

(f)

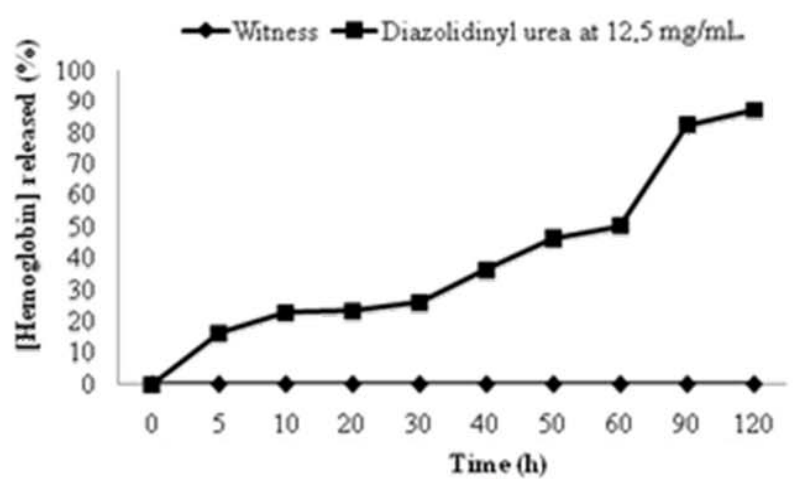

(h)

Fig. 2 Release of hemoglobin by erythrocytes induced by different concentrations of Diazolidinyl urea (a) 0.097, (b) 0.195, (c) 0.39, (d) 0.781, (e) 1.562, (f) 3.125, (g) 6.25 and (h) $12.5 \mathrm{mg} / \mathrm{mL}$. 
The DU product consists of several compounds with presumably 1-(3,4-bis-hydroxymethyl-2,5dioxo-imidazolidin-4-yl)-1, 3-bis-hydroxymethyl-urea (compound BHU) as dominant. The content of BHU on behalf of the relative response factor of $\mathrm{HU}$ is estimated to be $3 \%-40 \%$. The remaining part of DU is presumably numerous polymers of allantoin formaldehyde condensation products [9].

Just as imidazolidinyl urea, we note that the diazolidinyl urea-induced hemolysis in two phases, the first phase of 10-40 min of incubation. The second phase was after 60 to $120 \mathrm{~min}$ of incubation. It seemed to us the imidazolidinyl urea, that diazolidinyl urea also showed its activity through the release of formaldehyde in the first stage and by its parent molecules in the second stage.

Perhaps the results of the current investigation would serve as a starting point for such additional studies.

\section{Conclusions}

In conclusion, IU and DU have to be considered as erythrocyte cytotoxic in vitro. It appeared from this work that the concentrations allowed in cosmetic products induced the release of hemoglobin from red cells to the rate of: $33 \%-36 \%$ for imidazolidinyl urea, $75 \%-82 \%$ for diazolidinyl urea after $120 \mathrm{~min}$ of incubation.

The clear erythrocyte cytotoxic seems to be caused by formaldehyde released or by the action of the parent chemical structure of the compounds. A re-evaluation of the risk assessment of these compounds seems warranted and should take into account results from the existing database.

\section{References}

[1] M. Bason, V. Gordon, H. Maibach, Skin irritation-in vitro assays, Int. J. Dermatol. 30 (1991) 623-626.

[2] J.C. Davila, R.J. Rodriguez, R.B. Melchert, D.Jr. Acosta, Predictive value of in vitro model systems in toxicology, Annu. Rev. Pharmacol. Toxicol. 38 (1998) 63-96.

[3] W. Gfeller, W. Kobel, G. Seifert, Overview of animal test methods for skin irritation, Food Chem. Toxicol. 23 (2) (1985) 165-168.

[4] P. Knox, P.F. Uphill, J.R. Fry, J. Benford, M. Balls, The Fram multicentre project on in vitro cytotoxicology, Food Cosmet. Toxicol. 24 (1986.) 457-463.

[5] L.S. Birnbaum, W.S. Stokes, Safety testing: Moving toward alternative methods, Environ Health Perspect 118 (1) (2010) A12-A13.

[6] P.A. Botham, L.K. Earl, J.H. Fentem, R. Roguet, J.J.M. van de Sandt, Alternative methods for skin irritation testing: The current status, Alternatives to Laboratory Animals (ATLA) 22 (1998) 195-211.

[7] D.E. Cadwallader, Behavior of erythrocytes in various solvent systems: 1. Water-glycerin and water-propylene glycol, J. Pharm. Sci. 52 (12) (1963) 1175-1180.

[8] W.J. Husa, J.R. Adams, Isotonic solutions: 2. The permeability of red corpuscles to various substances, J. Am. Pharm. Assoc. 33 (1944) 329.

[9] S.V. Lehmann, U. Hoeck, J. Breinholdt, C.E. Olsen, B. Kreilgaard, Characterization and chemistry of Imidazolidinyl urea and diazolidinyl urea, Contact Dermatitis. 54 (2006) 50-58.

[10] CTFA (Cosmetic Toiletry and Fragrance Association), Cosmetic Ingredient Dictionary, 1st ed., Washington, 1973, p. 232.

[11] F. Mari-Ann, Preservatives in registered chemical products, Contact Dermatitis. 53 (1) (2005) 27-32.

[12] 76/768/CEE, Council Directive of 27 July 1976 on the approximation of the laws of the Member States relating to cosmetic products (76/768/EEC), OJ L 262, 27.9.1976, p. 169. 1976L0768 EN 01.03.2010 027.0014.

[13] P.A. Berke, W.E. Rosen, Germall. 2: New broad-spectrum cosmetic preservative, Cosmet. Toilet. 97 (1982) 49-53.

[14] G.R. Kantor, J.S. Taylor, J.L. Ratz, P.L. Evey, Acute allergic contact dermatitis from diazolidinyl urea (Germall 2) in a hair gel, J. Am. Acad. Dermatol. 13 (1985) 116-119.

[15] M.G. DeNavarre, S.A. Freigert, The Chemistry and Manufacture of Cosmetics, Fla, Continental Press Orlando, 1975, pp. 332-353.

[16] R.L. Decker, J.A. Wenninger, Frequency of preservative use in cosmetic formulas as disclosed to FDA-1982 update, Cosmet. Toilet. 97 (1982) 57-59.

[17] R.E. Elder, Final report on the safety assessment for imidazolidinyl urea, J. Environ. Pathol. Toxicol. 4 (1980) 5-17.

[18] Cosmetic Ingredients Review Expert Panel (CIREP), Annual review of cosmetic ingredient safety assessments: 2001/2002, Int. J. Toxicol. 22 (Suppl. 1) (2003) 1-35.

[19] C. Anselmi, A. Ettorre, M. Andreassi, M. Centini, P. Neri, A.D. Di Stefano, In vitro induction of apoptosis vs. 
necrosis by widely used preservatives: 2-phenoxyethanol, a mixture of isothiazolinones, imidazolidinyl urea and 1,2-pentanediol, Biochem. Pharmacol. 63 (2002) 437-453.

[20] O.W. Robert, M. Vorapann, S. Mongkol, Determination of diazolidinyl urea in a topical cream by high performance liquid chromatography, J. Chromatogr. B. 696 (1997) 303-306.

[21] M.C. Jacobs, I.R. White, R.J. Rycroft, N. Taub, Patch testing with preservatives at St. John's from 1982 to 1993, Contact Dermatitis 33 (1995) 247-254.

[22] A. Schnuch, J. Geier, W. Uter, P.J. Frosch, Patch testing with preservatives, antimicrobials and industrial biocides, Results from a multicentre study, Br. J. Dermatol. 138 (1998) 467-476.

[23] Cosmetic Ingredient Review Expert Panel (CIREP), Annual review of cosmetic ingredient safety assessments: 2005/2006, Int. J. Toxicol. 27 (Suppl. 1) (2008) 77-142.

[24] M.A. Liebert, Final report on the safety assessment of diazolidinyl urea, J. Am. Coll. Toxicol. 9 (1990) 229-245.

[25] J. Bolard, How do the polyene macrolide antibiotics affect the cellular membrane permeability, Biochim. Biophys. Acta. 864 (1986) 258-303.

[26] T. Bock, B.W. Müller, A novel assay to determine the hemolytic activity of drugs incorporated in colloidal carriers systems, Pharm. Res. 11 (1994) 589-591.

[27] W.J.W. Pape, U. Pfannenbecker, U. Hoppe, Validation of the red blood cell test system as in vitro assay for the rapid screening of irritation potential of surfactants, Mol. Toxicol. 1 (1987) 525-536.

[28] O. Reer, T. Bock, B.W. Müller, In vitro corneal permeability of diclofenac sodium in formulations containing cyclodextrins compared to the commercial product voltaren ophtha, J. Pharm. Sci. 83 (1994) 1345-1349.

[29] Scientific Committee on Cosmetic Products and Non-Food Products intended for consumers (SCCNFP), A clarification on the formaldehyde and para-formaldehyde entry in directive 76/768/EEC on cosmetic products, SCCNFP/587/02 (2002).

[30] G. McDonnell, A.D. Russell, Antiseptics and disinfectants: activity, action, and resistance, Clin. Microbiol. Rev. 12 (1) (1999) 147-179. 